\title{
DEVELOPMENT AND SEGMENTATION OF VISUALLY CONTROLLED MOVEMENT BY SELECTIVE EXPOSURE DURING REARING ${ }^{1}$
}

\author{
ALAN HEIN,"2 RICHARD HELD, AND ELLEN C. GOWER \\ Massachusetts Institute of Technology
}

\begin{abstract}
Three experiments are reported which clarify the role of movement-produced changes in visual stimulation for the acquisition of visually controlled behaviors. The first study shows that exposure during passive transport, which provides an asystematic relation between self-produced movements and visual stimulation, delays the acquisition of visual-motor coordination when a kitten is subsequently free to locomote in light. The second experiment demonstrates that control of movement by one eye develops if that eye is exposed during locomotion. This control does not transfer to the contralateral eye which is exposed only during passive transport. In the final experiment, view of the forelimbs is restricted to one eye. Visually guided reaching develops under the control of that eye but does not transfer to the eye which has not viewed the limbs. The results of Experiments 2 and 3 provide evidence that the system for visual guidance of movement consists of components which can be acquired independently.
\end{abstract}

Movement during exposure to patterned light has been recognized as important for the development and maintenance of visually guided behaviors in higher mammals by Hebb (1949), Hein (1968), Held (1961), Riesen (1958), and others. One set of observations consistent with this premise derives from experiments in which animals are deprived of patterned light stimulation. Rearing in the dark or in diffused light produces substantial deficits in visually controlled behavior (Ganz \& Fiteh, 1968; Riesen, Kurke, \& Mellinger, 1953; Wiesel \& Hubel, 1965a). Although neural dysfunction has been observed in these animals (Ganz, Fitch, \& Satterberg, 1968; Wiesel \& Hubel, 1965b), there is evidence to suggest that visual-motor deficits are not due solely to abnormalities of the visual system (Ganz \& Fitch, 1968; Meyers \& McCleary, 1964; Wiesel \& Hubel, 1965a). Whatever damage to the

${ }^{1}$ This research was supported by Grants GB2728 and GB-6712 from the National Science Foundation and Grant NGL-22-009-308 from the National Aeronautics and Space Administration. The authors are grateful to Rhea Diamond for her help in the preparation of this manuscript.

${ }^{2}$ Requests for reprints should be sent to Alan Hein, Department of Psychology, Massachusetts Institute of Technology, Cambridge, Massachusetts 02139 . visual system results from patterned light deprivation, exposure to patterned light does not by itself assure normal development of visually controlled behavior. Direct evidence is provided by experiments in which neonates are either free to locomote in an intermittently illuminated environment or provided with patterned light stimulation only when prevented from moving. These animals fail to develop visually guided behaviors (Hein, Gower, \& Diamond, 1970).

Hein and Held (1962) suggested that the mechanism which underlies acquisition of visually guided behaviors utilizes the correlation between self-produced movement and change in visual stimulation. In an experimental examination of this hypothesis, kittens that locomoted in patterned light were compared with kittens passively transported within the same environment (Held \& Hein, 1963). When tested, the animals that had locomoted showed visual placement of their forelimbs and discrimination of deep from shallow sides of a visual cliff, but their passively transported littermates did not. After considering a number of alternative explanations, the authors concluded that variation in visual stimulation concurrent with and systematically dependent upon 
self-produced movement is the essential condition for development of visually guided behaviors. Since that time certain implications of this hypothesis have been experimentally investigated and summarized in the literature (Hein, 1970; Held, 1968a). In the present paper the authors report three of these studies in detail and examine more closely the contribution of motor-visual feedback to the development of visually coordinated behavior. Experiment 1 examines the effects of prior exposure during passive transport upon subsequent acquisition of visually controlled behavior during locomotion. Fixperiment 2 examines the effects of exposing one eye during locomotion and the other eye during passive transport. Experiment 3 determines if the development of visually guided reaching, which requires view of the limbs, can be acquired independently by each eye.

\section{EXPERIMENT 1}

Although deficient in visual placing and cliff discrimination, passively transported kittens show pupillary reflexes, visualcentering responses, tactual responses, and other motor activities immediately following exposure in a gondola (Held \& Hein, 1963). When the transported animals are removed from the gondola and permitted free locomotion in patterned light, they rapidly acquire visually controlled behaviors. These observations imply that passive transport neither induces general debility of the motor system nor results in deterioration of peripheral parts of the visual system. Held and Hein (1963) have suggested that deficits in the passively transported animals result from deprivation of the motor-visual feedback essential for development of visually controlled behaviors. Alternately, visually controlled behaviors may develop during passive transport but at a much slower rate than during locomotion. However, the finding that prolonged transport $(\mathbf{1 2 6} \mathrm{hr}$.) did not result in acquisition of visually triggered extension of the limbs or discrimination on a visual cliff argues against this alternative. It remains possible that the later development of visual-motor coordination is either facilitated or impeded by passive transport. The present experiment examines the effect of prior transport on the time required for development of visually controlled behaviors during subsequent locomotion.

\section{Method}

Exposure apparatus. The carousel apparatus described by Held and Hein (1963) was used to control movements and visual experience of young kittens. The animals were held with neck yokes and halters at opposite ends of a lever pivoted at its center. The lever and appropriate mechanical linkages transferred the movements of the locomoting kitten to the kitten transported in a gondola. Symmetry of the visible surround provided both animals with a similar view. Visual stimulation was systematically related to self-produced movements for the locomoting animals. For the transported animals, the relation between selfproduced movements and visual stimulation was asystematic, having been perturbed by movements of the gondola.

Subjects. Immediately after birth, 19 kittens were placed with their mothers and littermates in large cages within a totally darkened room. Thereafter they remained in the dark until termination of the experiment except during periods of exposure in the carousel apparatus and during testing of visual-motor capacities.

Procedure. When the experimental kittens were 8 wk. of age they were removed daily from the darkroom and placed in the gondola for $3 \mathrm{hr}$. of exposure in patterned light. A kitten of the same age locomoted at the other end of the lever. Its movements were transformed by the apparatus into movements of the gondola. This procedure was repeated for 11 days, providing $33 \mathrm{hr}$. of passive transport in light for the experimental animals-3 hr. more than the average duration of locomotion found sufficient for development of visually guided behaviors in the previous study. At the end of Hour 33, the experimental kittens were removed from the gondola, the vibrissae were cut, and the kittens tested for visual placing. Although forelimb extension could be elicited by vestibular or tactual stimulation, none of the passively transported animals extended their limbs on approaching a broad surface. The present authors now refer to this response as visually triggered extension. As previously emphasized (Held \& Hein, 1963), this extension response provides a test of visual-motor capacity which does not require prior training and hence does not confound exposure with testing. Only a technique that meets this requirement can reveal deficits which may be readily overcome during training.

On the day following testing, the animals were returned to the apparatus but placed at the opposite end of the lever and permitted to locomote for $3 \mathrm{hr}$. A metal bar equal in weight to the kitten was placed in the gondola. This exposure was repeated daily until the end of the experiment. After 
each exposure period, the animals were tested for visually triggered extension. The first six kittens used in this experiment were also observed during eight descents from the center platform of a visual cliff on the day the extension response was first displayed.

\section{Results}

All animals eventually acquired the extension response. The average duration of locomotion necessary for this development was $41 \mathrm{hr}$. In the original study, kittens of comparable age that had no prior exposure in light required a mean of $30 \mathrm{hr}$. to acquire extension (Held \& Hein, 1963). Figure 1 presents the distribution of durations of locomotion to acquire the extension response for the original group of locomoting kittens and for the group passively transported prior to locomotion. A MannWhitney $U$ test used to assess the significance of the difference between these groups yielded $p<.05$ (two-tailed).

The six kittens tested on the visual cliff were able to discriminate the shallow from the deep side. All descents were to the shallow side for five animals; one animal descended once to the deep side and seven times to the shallow.

\section{Discussion}

The present experiment reveals that prior passive transport delays acquisition of visually triggered extension and visual-cliff discrimination during subsequent locomotion. If passive transport simply deprives the animal of feedback essential for development of coordinated visualmotor behaviors, no clelay would be expected. The delay of acquisition during subsequent locomotion indicates that exposure with asystematic feedback, as provided by transport in the gondola, has some special disruptive effect.

Another piece of evidence consistent with this interpretation is the observation that passively transported animals do not exhibit visually triggered extension. This response has been shown to develop with exposure in light, in the absence of view of the limbs (Hein \& Held, 1967), and, indeed, in the absence of any motor-visual feedback (Hein et al., 1970). The failure of kittens transported in the gondola to

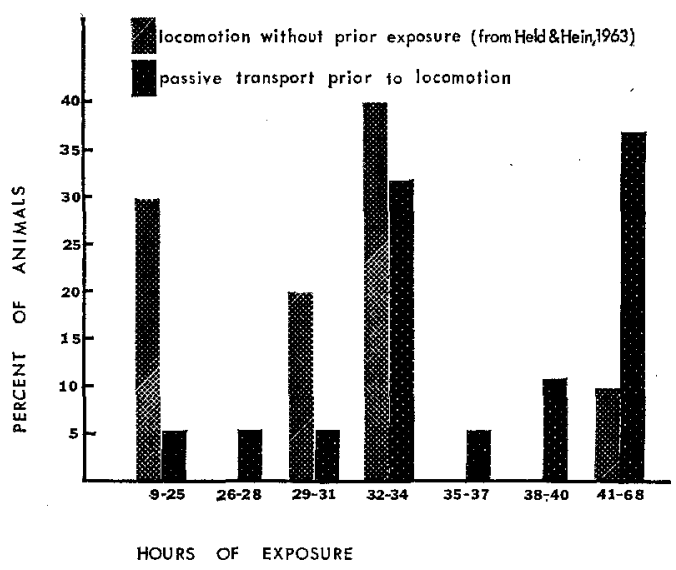

Frg. 1. Number of hours of exposure during locomotion necessary to acquire extension response in groups with and without prior passive transport.

show the extension response can therefore be interpreted as a specific effect of exposure with asystematic motor-visual feedback.

\section{EXPERTMENT 2}

Held and Hein (1963) argued that neither disruption of performance by shock, fright, or overactivation upon release from the deprived state, nor the development of competing responses, could account for the deficits which follow passive transport. Such behaviors were not seen during observation of their experimental animals at the end of the exposure period. At that time the kittens that had been passively transported displayed appropriate pupillary and pursuit reflexes and tactual-placing responses. This suggests that the deficits of passively transported animals do not reflect atrophy of the visual system or debility of the motor system. However, it is possible that these animals fail to perform visual-placing responses and visual-cliff discriminations due to generalized inhibition of movement in response to visual stimuli. This possibility may be excluded by showing that the passively transported kitten performs visually controlled movements under certain conditions. The attempt to meet this requirement involves the exposure of each eye under a different condition. Riesen et 
TABLE 1

Dhacents from Visual Cliff following AlterNating Monocular Exposure during Locomotion and Passive Transport

\begin{tabular}{|c|c|c|c|}
\hline \multirow[b]{2}{*}{ Subject } & \multirow{2}{*}{$\begin{array}{l}\text { Eye exposed } \\
\text { during } \\
\text { locomotion }\end{array}$} & \multicolumn{2}{|c|}{ Cliff descents (shallow/deep) } \\
\hline & & $\begin{array}{l}\text { Eye exposed } \\
\text { during } \\
\text { locomotion }\end{array}$ & $\begin{array}{l}\text { Ey e exposed } \\
\text { during } \\
\text { transport }\end{array}$ \\
\hline 1 & $\mathrm{R}$ & $8 / 0$ & $5 / 3$ \\
\hline 2 & $\mathrm{~L}$ & $8 / 0$ & $2 / 6$ \\
\hline 3 & L & $8 / 0$ & $4 / 4$ \\
\hline 4 & $\mathrm{~L}$ & $7 / 1$ & $6 / 2$ \\
\hline 5 & $\mathrm{R}$ & $7 / 1$ & $5 / 3$ \\
\hline 6 & $\mathrm{~L}$ & $8 / 0$ & $5 / 3$ \\
\hline Total & & $46 / 2$ & $27 / 21$ \\
\hline
\end{tabular}

al. (1953) and Chow and Nissen (1955), using a similar procedure, found deficits in interocular transfer of discriminations learned monocularly. Their results suggested that exposing one eye during locomotion and the other during passive transport might produce an animal which showed visually controlled responses when tested with one eye but not when tested with the other.

\section{Method}

Subjects. Immediately after birth, six kittens were placed in the dark with their mothers and littermates and remained in darkness except during periods of controlled exposure in light and testing.

Procedure. Beginning when they were 4 wk. of age, the kittens received $3 \mathrm{hr}$. of exposure daily. When a kitten was removed from the dark, an opaque vinyl oceluder was fitted over the sclera. and under the lid of one eye. The animals were run in pairs. One kitten was passively transported in a gondola at one end of the levered arm while the other was free to locomote at the other end. After $1.5 \mathrm{hr}$. of exposure in light, the occluder was transferred to the contralateral eye and the positions of the two animals exchanged. This exposure was continued for 1.5 additional hr. Thus, periods when visual stimulation of one eye accompanied self-produced movement alternated with periods when visual stimulation was provided to the other eye during passive transport. The kittens were tested monocularly after each $1.5 \mathrm{hr}$. of exposure for presence of the extension response. Scoring was done by an observer who did not know the exposure history of the animal. On the day that the kitten first showed triggered extension, using either eye, it was tested monocularly with each eye for discrimination on the visual cliff.

\section{Results}

The results of this experiment are summarized in Table 1. When using the eye exposed during locomotion, all kittens eventually showed both visually triggered extension to a broad horizontal surface and a preference for the shallow. side of the visual cliff. When using the eye exposed during passive transport, no kitten showed the extension response and all failed to discriminate between the shallow and deep sides of the visual cliff. A sign test of the difference between number of descents to the shallow side when using the actively vs. passively exposed eye is significant at $p<.03$ (one-tailed).

\section{Discussion}

When using the eye exposed during locomotion, the kitten displays visually controlled behaviors. When using the eye exposed only during passive transport, these responses are absent. Since the animal does respond appropriately to visual stimuli, generalized response inhibition cannot account for deficits resulting from passive transport.

The results of Experiments 1 and 2 when taken together with the report of Hein et al. (1970) indicate that each condition of exposure may support the development of certain capacities and not others. Animals that have been dark-reared show no visually controlled behavior except pupillary reflexes and visual-centralizing responses. Rearing in diffused light allows the triggered component of the visualplacing response to develop, while visually guided reaching and visually guided locomotion do not. Exposure of an immobilized kitten in a normally illuminated environment or exposure of a freely locomoting kitten in a stroboscopically illuminated environment will not support development of visually guided behaviors, although the visually triggered extension response is acquired. Visually guided behaviors develop only when the exposure conditions provide opportunity for self-produced movement systematically associated with changes in visual stimulation. When selfproduced movements are asystematically 
associated with changes in visual stimulation neither visually guided behaviors nor the visually triggered extension response develop.

The demonstration that the development of visual-motor coordination is displayed only when using the eye exposed during locomotion has a further implication. Control of behavior by each eye is identified as an independent component of visual-motor coordination. Acquisition of each component requires exposure of that eye with visual feedback from self-produced movements. This finding encouraged the search for other independent compoments of visual-motor coordination.

\section{EXPERIMENT 3}

Hein and Held (1967) have shown that visually controlled placing of the forelimbs has both triggered and guided components. Only the triggered component developed when kittens locomoting in light were prevented from viewing their limbs. These animals displayed the extension response to a broad surface but could not guide their limbs to the position of a small target in visual space. Failure to develop visually guided reaching was not accompanied by deficits in the use of the limbs for visually guided locomotion. The kittens moved about efficiently; they avoided obstacles and were indistinguishable from normal animals as they jumped on and off various objects. It was concluded that use of the limbs in visually guided locomotion and visually guided reaching by those same limbs constitute two independent components of visual-motor coordination.

The present. experiment determined whether control of visually guided reaching might be acquired independently by each eye. This possibility was examined by permitting view of the limbs by one eye but not the other.

\section{Method}

Subjects. Of the 12 kittens which served as subjects for this experiment, 10 were dark-reared until they were $5-12$ wk. of age and then exposed in light for $6 \mathrm{hr}$. a day. The remaining two kittens were reared from wirth in a room illuminated for 24 hr. a day.

Procedure. The animals that had been dark- reared were permitted to locomote freely in light for $6 \mathrm{hr}$. a day while wearing lightweight plastic collars. For $3 \mathrm{hr}$. they wore opaque collars which prevented view of the limbs and torso following the procedure described by Hein and Held (1967). For the remaining $3 \mathrm{hr}$. they wore clear collars of the same size and weight which permitted view of the limbs. Three different exposure conditions were used. In Condition A, five kittens had one eye occluded while the opaque collar was worn, and other eye occluded while the clear collar was worn. In Condition B, five kittens had both eyes open while the opaque collar was worn, and one eye occluded while the clear collar was worn. Except for these exposure periods, all animals of Conditions $A$ and $B$ were kept without collars in total darkness. In Condition $\mathrm{C}$, two kittens were fitted at birth with opaque collars large enough to prevent view of the limbs without interfering with either locomotion or suckling. These animals locomoted freely in light for $24 \mathrm{hr}$. a day. As the kittens grew, successively larger collars were provided so that view of the limbs remained unavailable. When the animals were 30 days old, the opaque collars were replaced by clear collars for $3 \mathrm{hr}$. each day, during which time one eye was occluded.

The dark-reared kittens were exposed under Condition A or Condition B for 5,6 , or 7 days before being tested for visually guided reaching. The two kittens of Condition $\mathrm{C}$ were exposed with monocular view of the limbs for 17 and 21 days, respectively, before testing. The animals were tested monocularly, first with one eye and then with the other.

The apparatus used for measuring visually guided reaching is a board with spaced cutouts toward which the animal is lowered (described by Hein \& Held, 1967). The discontinuous edge is formed by prongs $20.0 \mathrm{~cm}$. long and $2.5 \mathrm{~cm}$. wide separated by $7.5 \mathrm{~cm}$. A test consisted of 12 trials, 6 with each forelimb. When the animal extended a limb, contact of one or more claws with the top of a prong was scored as a hit. Testing and scoring were done by observers who did not know the exposure history of the animal.

\section{Results}

The results of Experiment 3 are presented in Table 2. The three conditions do not appear to differ in their effects upon development of guided reaching. When using the eye which had previously viewed the limbs, all kittens showed visually guided reaching. When using the eye which had not previously viewed the limbs, the percentage of hits was not significantly different from chance. A sign test of the difference in percentage of hits is significant at $p<.001$ (one-tailed). 
TABLE 2

Guided Reaching afTer Expostre with MoNOCULAR VIEW OF THE LimbS

\begin{tabular}{l|r|r|r}
\hline Condition & Subject & $\begin{array}{c}\text { Eye with } \\
\text { view of } \\
\text { limbs (hits/ } \\
\text { misses) }\end{array}$ & $\begin{array}{c}\text { Eye without } \\
\text { view of } \\
\text { limbs (hits } \\
\text { misses) }\end{array}$ \\
\hline A: Alternating mo- & 1 & $11 / 1$ & $5 / 7$ \\
nocular & 2 & $12 / 0$ & $8 / 4$ \\
& 3 & $11 / 1$ & $7 / 5$ \\
& 4 & $10 / 2$ & $5 / 7$ \\
B: Alternating mo- & 6 & $12 / 0$ & $5 / 7$ \\
nocular-binocular & 7 & $12 / 0$ & $7 / 5$ \\
& 8 & $11 / 1$ & $8 / 4$ \\
& 9 & $11 / 1$ & $7 / 5$ \\
C: Prolonged binocu- & 11 & $12 / 0$ & $6 / 6$ \\
lar & 12 & $12 / 0$ & $7 / 5$ \\
All conditions & & $11 / 1$ & $5 / 7$ \\
\hline
\end{tabular}

\section{Discussion}

Visually guided reaching is acquired only if an animal is permitted to view the movements of its limbs (Hein \& Held, 1967). In Condition A, exposure of one eye with view of the limbs alternated with exposure of the contralateral eye without view of the limbs. Accurate reaching when using one eye indicates that appropriate movements are available to visual control. The absence of visually guided reaching when using the contralateral eye implies that each eye must view the limbs to acquire this capacity. The results of Condition $\mathrm{B}$ demonstrate that binocular exposure to the environment without view of the limbs does not obviate this requirement. Condition $\mathrm{C}$ explored the possibility that continuous binocular exposure during the neonatal period might facilitate interocular transfer. However, control of visually guided reaching remained confined to the eye that had viewed the limbs.

Normally, control over visually guided behavior is acquired concurrently by the two eyes. This coincidence has obscured the possibility for independent acquisition of control by each eye, here demonstrated by failure of guided reaching to transfer interocularly under all three conditions in which the two eyes were dissociated with: respect to view of the limbs.

\section{General Discussion}

The first experiment showed that passive transport not only fails to support development of visually controlled behaviors but also delays subsequent acquisition of these capacities during locomotion. This delay indicates that passive transport has a special disruptive effect upon the development of visual-motor coordination. Other evidence for this effect was adduced from the failure of passively transported animals to show visually triggered extension of the forelimbs. The extension response has been demonstrated to develop under exposure conditions which do not provide visual feedback (Hein et al., 1970). This series of findings suggests that exposure during passive transport has two distinct effects. (a) The deprivation of systematic motor-visual feedback precludes accurate visually guided behavior. This conforms to the original hypothesis that the acquisition of guided behavior depends upon the correlation of self-produced movement with variation in visual stimulation. (b) The asystematic relation between movement and visual stimulation results in the failure to develop visually triggered extension of the forelimbs.

The second experiment demonstrated that control over visual placing and visually guided locomotion, gained by an eye exposed during locomotion, is not shown by the contralateral eye exposed only during passive transport. The present authors interpreted this result as evidence that the deficits which result from exposure with passive transport are not attributable to generalized inhibition of response to visual stimuli. In addition, it was concluded that control of locomotion by each eye constitutes an independent component of visually coordinated behavior.

In the third experiment, control of visually guided reaching was acquired only by the eye which had viewed the limbs. This result identifies mediation of guided limb movements by each eye as an 
independent component of visual-motor coordination.

This series of studies has utilized the experimental technique of selective exposure to serve two purposes. (a) It has identified certain independent components of visually coordinated behavior; and (b) it has specified the conditions essential for their acquisition. Each component of visually guided behavior identified here develops only with appropriate feedback from self-produced movement. The present authors suggest that integration of these components during the neonatal period permits the achievement of normal visualmotor coordination.

A surprising outcome of these studies is the lack of interocular equivalence found in Experiments 2 and 3. This result contrasts with the report of interocular transfer of form discrimination in kittens reared without patterned visual stimulation (Meyers \& McCleary, 1964). The presence or absence of interocular equivalence has been used to identify two alternate modes of processing visual-spatial informationone for responses guided to the location of objects in space and the other for identification of certain figural properties of those objects (Held, 1968b). Lack of interocular transfer of control over visually guided movement is consistent with that distinction.

\section{REFERENCES}

CHow, K. L., \& Nissen, H. W. Interocular transfer of learning in visually naive and experienced chimpanzees. Journal of Comparative and Physiological Psychology, 1955, 48, 229-237.

Ganz, L., \& Fitch, M. The effect of visual deprivation on perceptual behavior. Experimental Neurology, 1968, 22, 638-660.

Ganz, L., Fitch, M., \& SatTerberg, J. A. The selective effect of visual deprivation on receptive field shape determined neurophysiologically. Experimental Neurology, 1968, 22, 614-637.

HEBB, D. O. The organization of behavior. New York: Wiley, 1949.

HeTN, A. Labile sensorimotor coordination. In J. Gordon (Ed.), Malnutrition, learning, and be- havior. Cambridge: Massachusetts Institute of Technology Press, 1968.

HeIN, A. Recovering spatial-motor coordination after visual cortex lesions. In D. A. Hamburg, K. H. Pribram, \& A. J. Stunkard (Eds.), Perception and its disorders. Baltimore: Williams \& Wilkins, 1970.

Hein, A., Gower, E. C., \& Diamond, R. M. Exposure requirements for developing the triggered component of the visual-placing response. Journal of Comparative and Physilogical Psychology, 1970, 73, 188-192.

Hern, A., \& Her, , R. A neural model for labile sensorimotor coordination. In E. Bernard \& M. R. Kare (Eds.), Biological prototypes and synthetic systems. Vol. 1. New York: Plenum Press, 1962.

HeIn, A., \& Hexd, R. Dissociation of the visual placing response into elicited and guided components. Science, 1967, 158, 390-392.

HeLd, R. Exposure-history as a factor in maintaining stability of perception and coordination. Journal of Nervous and Mental Diseases, 1961, 132, 26-32.

HELD, R. Action contingent development of vision in neonatal animals. In D. P. Kimble (Ed.), Experience and capacity. New York: Academy of Sciences, 1968. (a)

Hewd, R. Dissociation of visual functions by deprivation and rearrangement. Psychologische Forschung, 1968, 31, 338-348. (b)

HeLD, R., \& HeIN, A. Movement-produced stimulation in the development of visually guided behavior. Journal of Comparative and Physiological Psychology, 1963, 56, 872-876.

MeYers, B., \& McCleary, R. A. Interocular transfer of a pattern discrimination in pattern deprived cats. Journal of Comparative and Physiological Psychology, 1964, 57, 16-21.

RIESEN, A. H. Plasticity of behavior: Psychological aspects. In H. F. Harlow \& C. J. Woolsey (Eds.), Biological and biochemical bases of behavior. Madison: University of Wisconsin Press, 1958.

Riesen, A. H., Kurke, M. I., \& Melifinger, J. D. Interocular transfer of habits learned monocuIarly in naive and visually experienced cats. Journal of Comparative and Physiological Psychology, 1953, 49, 166-172.

Wigsel, T. N., \& Hubet, D. H. Extent of recovery from the effects of visual deprivation in kittens. Journal of Neurophysiology, 1965, 28, 1060-1072. (a)

WiEser, T. N., \& Hubet, D. H. Comparison of the effects of unilateral and bilateral eye closure on cortical unit responses in kittens. Journal of Neurophysiology, 1965, 28, 10291040. (b)

(Received November 14, 1969) 